\title{
Probing the Oxidative Etching Induced Dissolution of Palladium Nanocrystals in Solution by Liquid Cell Transmission Electron Microscopy
}

Yingying Jiang, ${ }^{a}$ Guomin Zhu, ${ }^{a, \perp}$ Ganxing Dong, ${ }^{a}$ Fang Lin,,${ }^{b, a}$ Hui Zhang, ${ }^{a}$ Jun Yuan, ${ }^{c, a}$ Ze Zhang, ${ }^{a}$ and Chuanhong $\operatorname{Jin}^{a}$ *

a. State Key Laboratory of Silicon Materials, School of Materials Science and Engineering, Zhejiang University, Hangzhou, Zhejiang 310027, P. R. China. E-mail: chhjin@zju.edu.cn

${ }^{\mathrm{b}}$ College of Electronic Engineering, South China Agricultural University, Guangzhou, Guangdong 510642, P. R. China

${ }^{\text {c. }}$ Department of Physics, University of York, Heslington, York, YO10 5DD, United Kingdom

${ }^{\perp}$ Present address: Departments of Materials Science and Engineering, University of

Washington, Seattle, Washington 98195, United States

\begin{abstract}
A microscopic study of dissolution process of nanocrystals, an opposite while functioning cooperatively with growth in many cases, is an essential issues in variety aspects of research on nanocrystals. In this work, an in situ study of the dynamic dissolution process of palladium nanocrystals by liquid cell transmission electron microscope (TEM) is presented. The effective critical size $\left(R_{\text {critical }}\right)$ for monodispersed nanocrystals is determined to be about $5 \mathrm{~nm}$ in the experimental condition of this article. When the size of nanocrystal is above $R_{\text {critical }}$, the dissolution rate $(d r / d t)$ is nearly a constant. For the nanocrystal sizing below $R_{\text {critical }}$, the dissolution rate $(d r / d t)$ increases with the decrease of the nanocrystal radius $r$, indicating that high equilibrium solubility must be taken into account in the dissolution rate of small nanocrystals in solution. It is found that the aggregation kinetics and confinement effect between adjacent nanocrystals have effects on the dissolution rate during the reaction, and it has been analyzed in details and discussed in terms of the underlying physics involved. Lastly, the effects of electron beam-water interaction and the iron (III) agents on the oxidative etching are also compared.
\end{abstract}

Keywords: liquid cell TEM, in situ, oxidative etching, Pd nanocrystal 


\section{Introduction}

Solution-phase approach has emerged as a powerful and versatile tool for the synthesis of noble-metal nanocrystals with well-controlled size, shape, and structure in recent years (Sau and Murphy, 2004; Tao et al., 2008; Wang et al., 2005; Xia et al., 2009; Zhang et al., 2012). In a typical synthesis reaction, the growth and dissolution of nanocrystals are generally two cooperative but opposite processes: both of them can be the deterministic factor on achieving the final product. In comparison to the enormous progress in the structure-controlled synthesis of nanocrystals, the community have paid little attention to the dissolution process (Goto, 1958; Liu et al., 2008; Liu et al.; 2009).

Oxidative etching is one important type of dissolution processes, and it has been adopted as an effective and robust tool to tailor the compositions and structures of noble-metal nanocrystals (Zheng et al., 2014). In many cases, oxidative etchants such as bromide ion/oxygen $\left(\mathrm{Br}^{-} / \mathrm{O}_{2}\right)$, chloride ion/oxygen $\left(\mathrm{Cl}^{-} / \mathrm{O}_{2}\right)$, iron(II)/iron(III) $\left(\mathrm{Fe}^{2+} / \mathrm{Fe}^{3+}\right)$, or copper(I)/copper(II) $\left(\mathrm{Cu}^{+} / \mathrm{Cu}^{2+}\right)$ are introduced into the reaction solution, and then etch away those seeding nanocrystals with higher chemical reactivity who contains structural defects. As such single-crystalline nanocrystals become enriched in the final resultant products (Ma et al., 2010; Xiong et al., 2005; Xiong et al., 2005; Zhang et al., 2011; Zhang et al., 2015; Zheng et al., 2014). Nevertheless, by varying the relative concentrations of oxidative etchants, this method can also be applied on tuning the sizes of nanocrystals through oxidizing the initially formed seeds into precursors to redeposit on the remaining seeds. Given the considerations mentioned above understanding the dynamic evolution of oxidative etching in these syntheses is essential to accurately dictate the growth of nanocrystals, particularly at microscopic scale.

To address this issue, there have been considerable progress in revealing the dynamic evolution of oxidative etching and the mechanism behind, while most of them were conducted ex situ, i.e., taking liquid drops from the reacting solution at different stages for further characterizations (Ma et al., 2010; Xiong et al., 2005; Zhang et al., 2015; Zheng et al., 2014), 
during which the dissolution dynamics is not feasible due to the intrinsic technic limitations. The recent emergence tools, such as liquid cell TEM, has been successfully applied to probe the reaction dynamics in different applications including electrochemical reaction, nanocrystal growth and assembly, corrosion, oxidative etching, etc (Chee et al., 2014; de Jonge and Ross, 2011; Evans et al., 2011; Hermannsdörfer et al., 2015; Jiang et al., 2014; Liao and Zheng, 2016; Ross, 2015; Ross et al., 2016; Williamson et al., 2003; Wu et al., 2016; Zheng et al., 2009; Zeng et al., 2015).

In our previous report (Jiang et al., 2014), the oxidative etching of palladium nanocrystals was realized mainly due to generation of oxidation agents (such as $\mathrm{OH} \cdot, \mathrm{HO}_{2} \cdot, \mathrm{O}, \mathrm{H}_{2} \mathrm{O}_{2} \ldots$.) generated from the interaction between energetic electron beam and water solution (so-called radiolysis process), and the presence of bromide ions may further promote the dissolution of palladium by forming tetrabromopalladate ions $\left[\mathrm{PdBr}_{4}\right]^{2-}$.While, in this work, we adopted $\mathrm{Fe}^{3+}$, which is a common strong oxidative agent, as the etchant to study the dissolution kinetics of Pd nanocrystals, the results of which can be more correlated with ex situ etching results. We also chose TEM mode rather than STEM mode as the former one could typically offer wide distribution of electron illumination which would create a more homogenous reaction environment rather than STEM mode. Also, in our experimental condition used here, TEM mode has relative higher temporal resolution which should be more beneficial to gain dynamics and obtain detailed results, particularly for those nanocrystals sizing smaller than five nanometers where dissolution is too fast to be followed in the previous STEM observation (Jiang et al., 2014).

In this article, dissolution kinetics of single and aggregated $\mathrm{Pd}$ nanocrystals in $\mathrm{FeCl}_{3}$ aqueous solution was investigated directly through in situ liquid cell TEM. There exist a critical size $\left(R_{\text {critical }}\right)$ during the dissolution process below which the equilibrium solubility must be taken into account in the analysis of the dissolution rate. The aggregation kinetics and confinement effects for dissolution rate have been analyzed. In addition, the 
attraction/repulsion behavior of nanocrystals during dissolution has been discussed. These results are of great importance for dictating the controlled synthesis of nanocrystals with welldefined shapes and sizes.

\section{Experimental details}

Preparation of the Reaction Solution: All chemicals including poly (vinyl pyrrolidone) (PVP, MW $\approx 55000$, Aldrich), L-ascorbic acid (AA, Aldrich), sodium tetrachloropalladate $\left(\mathrm{Na}_{2} \mathrm{PdCl}_{4}\right.$, Aldrich), potassium bromide (KBr, Aladdin), potassium chloride (KCl, Aladdin), ferric chloride $\left(\mathrm{FeCl}_{3}\right.$, Aladdin) were used as received without further purification. $\mathrm{Pd}$ nanocubes (with an average edge length of $18 \mathrm{~nm}$ ) were prepared following a previous report (Jin et al., 2011) and washed with deionized water for five cycles to eliminate the residual reactants before loaded into the liquid cells for TEM observations. The reaction solution consisted of palladium nanocubes and $\mathrm{FeCl}_{3}$ aqueous solutions $(0.228 \mathrm{~mol} / \mathrm{L})$.

TEM Characterization: Liquid flow holder (Hummingbird Scientific Inc) was used for in situ experiments, on which two silicon chips with electron transparent silicon nitride windows (50 $\mathrm{nm}$ thickness) in the central area were sandwiched to encapsulate aqueous solution with confined thickness (around $500 \mathrm{~nm}$ ). In situ TEM experiments were carried out using a microscope (FEI Tecnai $\mathrm{G}^{2} \mathrm{~F} 20$ ) operated at $200 \mathrm{kV}$, the electron dose used here was in the range from 136 to 1020 electrons $/ \AA^{2}$.s. To further improve the attainable spatial resolution and contrast of TEM imaging, we adopted the "bubble" method to reduce the effective thickness of water layers inside the cell (Zhu et al., 2013). Ex situ characterization was conducted in spherical aberration corrected (Cs-corrrected) Chemi-STEM (FEI Titan). Software Camstudio, ImageJ and Gatan DigitalMicrograph were used to record and analyse the experimental TEM images.

\section{Results and discussion}


Pd nanocubes with an average edge length of $18 \mathrm{~nm}$ were loaded into the liquid cell holder

(Hummingbird Scientific Inc) for TEM observations. The reaction solution consisted of palladium nanocubes and $\mathrm{FeCl}_{3}$ aqueous solutions $(0.228 \mathrm{~mol} / \mathrm{L})$.
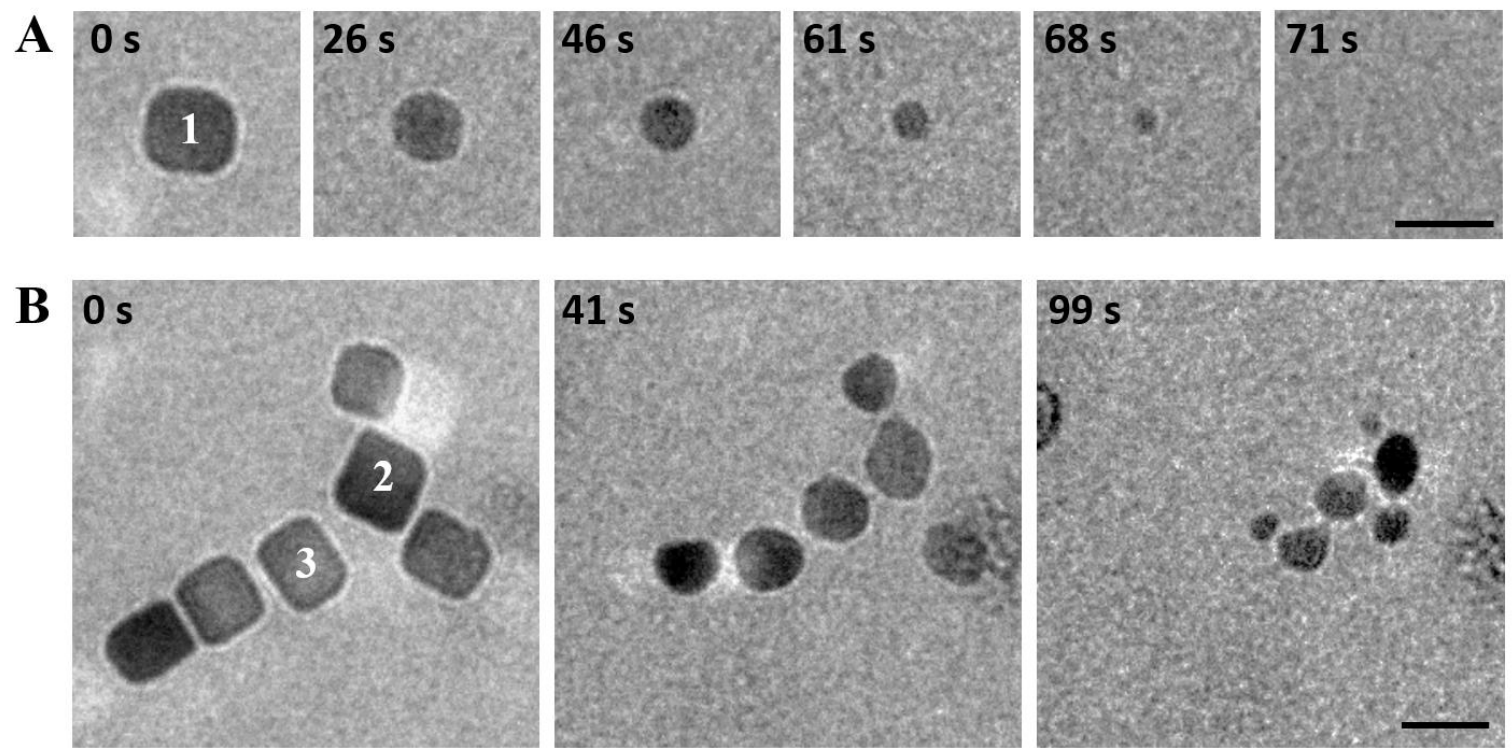

C
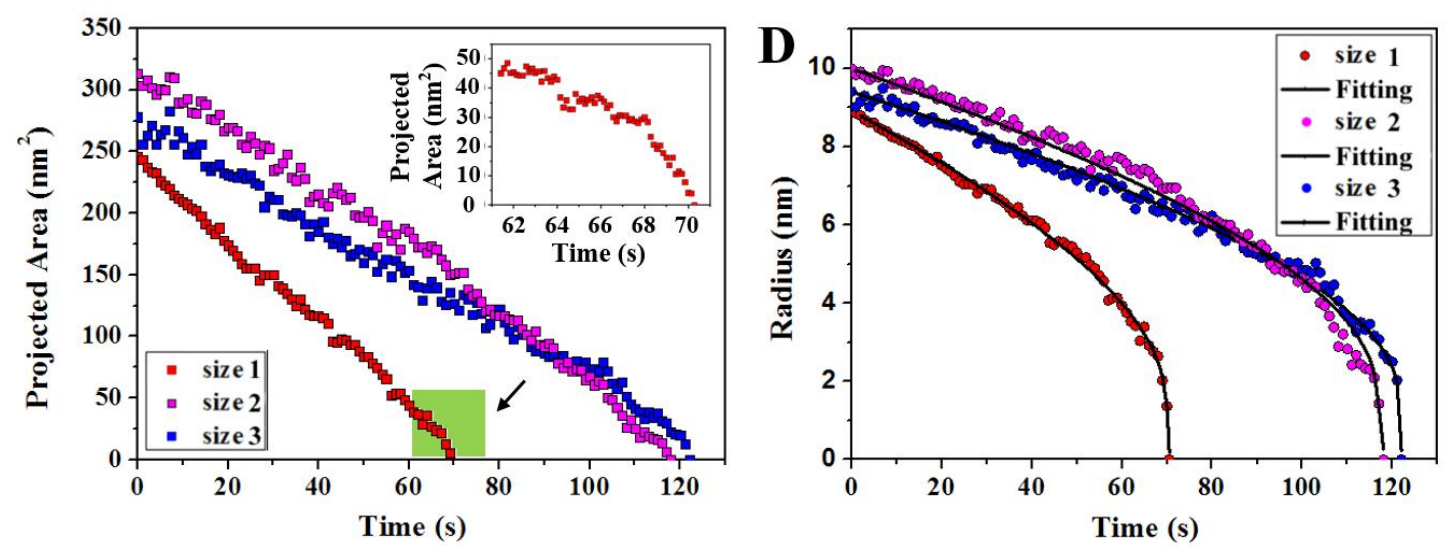

Figure 1. TEM images of in situ dissolution process for A) monodispersed and B) aggregated Pd nanocrystals. The electron dose used here is 136 electrons/ $\AA^{2}$. s. All scale bars are $20 \mathrm{~nm}$. C) Projected area and D) radius variations of the three marked Pd nanocrystals in A) and B) with reaction time.

TEM images shown in Figure 1A and 1B denote the size and morphology evolutions of monodispersed and aggregated Pd nanocrystals during the oxidative etching by the $\mathrm{Fe}^{3+}$, respectively. $\mathrm{As}^{3+} \mathrm{e}^{3+}$ ions function as oxidizing agents which can oxidize $\mathrm{Pd}$ atoms into 
$\left[\mathrm{PdCl}_{4}\right]^{2-}$ in the presence of $\mathrm{Cl}^{-}$ions arising from $\mathrm{FeCl}_{3}$ referring to the standard potentials $\left(E_{0}\right)$ of the redox reactions involved in the dissolution process (Weast, 1980).

$\mathrm{Fe}^{3+}+\mathrm{e}^{-} \rightarrow \mathrm{Fe}^{2+} \quad E_{0}=0.77 \mathrm{~V}$

$\left[\mathrm{PdCl}_{4}\right]^{2-}+2 \mathrm{e}^{-} \rightarrow \mathrm{Pd}+4 \mathrm{Cl}^{-} \quad E_{0}=0.59 \mathrm{~V}$

After carefully analysis over a large number of dissolving Pd cubes, we found that when the nanocrystal was isolated as illustrated in Figure 1A, the dissolving nanocrystals started from cubic structures. As the corners and edges dissolved faster than flat surfaces, the nanocubes evolved into spheres, then kept shrinking until the fully disappearance. While, when the nanocrystals were aggregated, as shown in Figure 1B, they were first pinned to their original positions for the first 41s. Accompanied with the shrinking sizes of the nanocrystals, most of them started to migrate and aggregate with others (see Figure 1B at 99 s, and also Movie S1).The viscous force decreases as the particle size decreases (Batchelor, 2000). In the case of small nanocrystals, the viscous force may not be strong enough to dampen down the random motion of Pd nanocrystals in the thin water layer, leading to their movement and aggregation.

The size evolution of three marked Pd nanocrystals in Figure 1A and 1B (marked as 1, 2, 3) with the reaction time was depicted in Figure 1C (projected area vs. time) and Figure 1D (radius vs. time). The projected area and radius was found to decrease with the reaction time, as shown in Figure 1C, similar with previous report (Liu et al., 2009). However, the better temporal resolution of the in situ TEM observation over the STEM observation reveals an interesting close-up view of the size evolution in the small size range (less than $5 \mathrm{~nm}$ ). Specifically, the radius decreases more rapidly when the size is smaller (Figure 1D). Goto et al. demonstrated that the law of the rate of dissolution $(d \mathrm{~m} / \mathrm{dt})$ only being proportional to the surface area did not hold when the size of the nanocrystals was smaller than $5 \mathrm{~nm}$ (Goto, 
1958). This can be interpreted as higher equilibrium solubility in smaller nanocrystals, and this parameter must be taken into account in the dissolution rate in solution (Iler, 1979).

Rate of dissolution $=d m / d t=K A S$

where $K$ is constant, related to the dissolution environment, and we assume the environment keeps the same during dissolution process, $A$ is the surface area of the nanocrystal, $S$ is solubility of the nanocrystal depending on the size, $m$ is mass of the dissolving nanocrystal at time $t$.

As $d m=\rho A d r$, where $\rho$ is density of the corresponding material, $r$ is radius of nanocrystal, so the dissolution equation (1) becomes:

$\rho A d r / d t=K A S$

$d r / d t=K S / \rho$

which means that the dissolution rate $(d r / d t)$ is a constant if the solubility of the nanocrystal remains the same. When the nanocrystal dissolves into smaller size, we used the modified version of the Kelvin equation (also known as the Gibbs-Thomson and as the OstwaldFreundlich equation) (Liu et al., 2008), which taken into account the effect of surface curvature in ultrasmall nanocryatals:

$S / S_{0}=\exp \left(R_{\text {critical }} / r\right)$

$R_{\text {critical }}=\left(2 \gamma V_{m}\right) /(R T)$

Where $S_{0}$ is the solubility of the bulk material, $V_{m}$ is the molecular volume, $\gamma$ is the surface free energy, $R$ is the gas constant, and $T$ is the temperature. So,

$d r / d t=K S_{0} \exp \left(R_{\text {critical }} / r\right) / \rho$

The differential function of equation (6) is found to be well matched with the data for radius vs. time of particle 1, as shown in Figure 1D. The fitted value of $R_{\text {critical }}$ is calculated to be $5.5 \mathrm{~nm}$ for particle 1 , which means that when the size of Pd nanocrystal decreased to critical size of around $5.5 \mathrm{~nm}$, the dissolution rate $(d r / d t)$ would have a sudden increase with the decrease of the radius of the nanocrystal. The value of $R_{\text {critical }}=5.5 \mathrm{~nm}$ could vary with 
different reaction conditions, such as molecular volume, surface free energy, reaction temperature, et al. This critical value was also observed in other systems such as during the evaporation of metal nanocrystals (Asoro et al., 2013). The size of evaporating nanocrystals also started to decrease drastically when it is below its $R_{\text {critical }}$.

From Figure 1C and 1D, it is shown that the dissolution rates of nanocrystal " 2 " and nanocrystal " 3 " are both smaller than that of particle " 1 ". It took almost twice the time for them to dissolve completely compared to the particle 1 (the average dissolution rates of nanocrystal 1, 2, and 3 are $0.12 \mathrm{~nm} / \mathrm{s}, 0.084 \mathrm{~nm} / \mathrm{s}$ and $0.077 \mathrm{~nm} / \mathrm{s}$ respectively). The different dissolution rate of these three nanocrystals might be caused due to different aggregation states since other conditions such as the concentration of $\mathrm{Fe}^{3+}$ ions, the electron dose, the thickness of liquid layer, and so on are almost kept the same. Compared to particle 2 and particle 3 surrounded by other particles, particle 1 was in a relatively separated state and was surrounded only by the reaction solution, lead to the faster dissolution rate. As a result, the dissolution rate is greatly influenced by the state of aggregation of nanocrystals. Further analyses of the influence of the aggregation states on the dissolution are detailed below. 

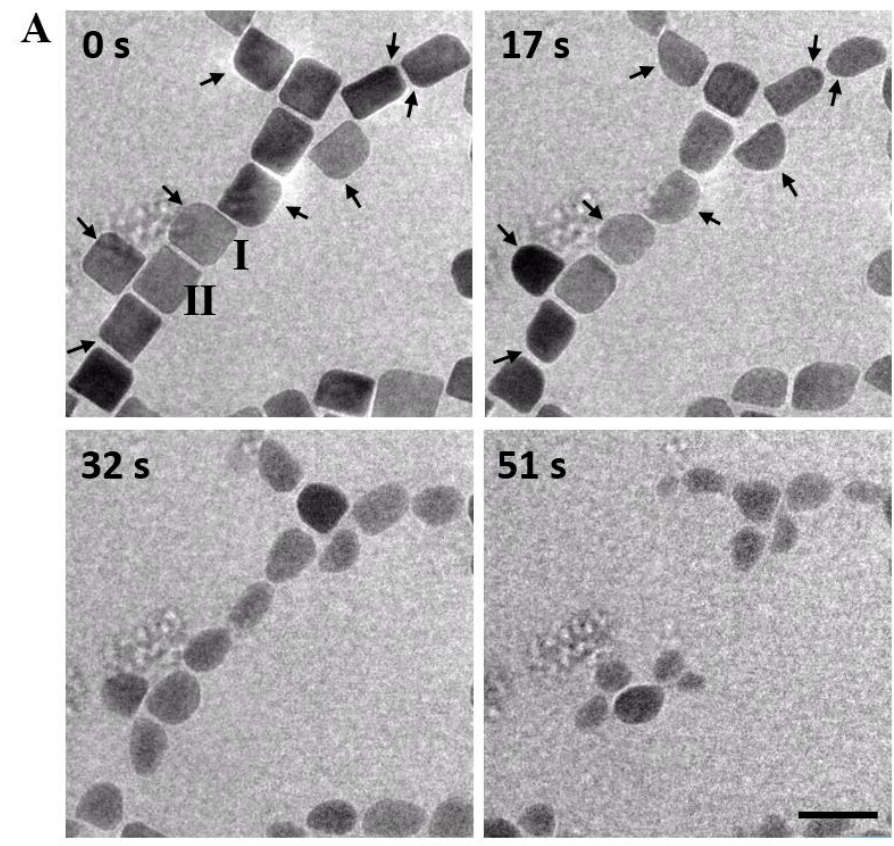
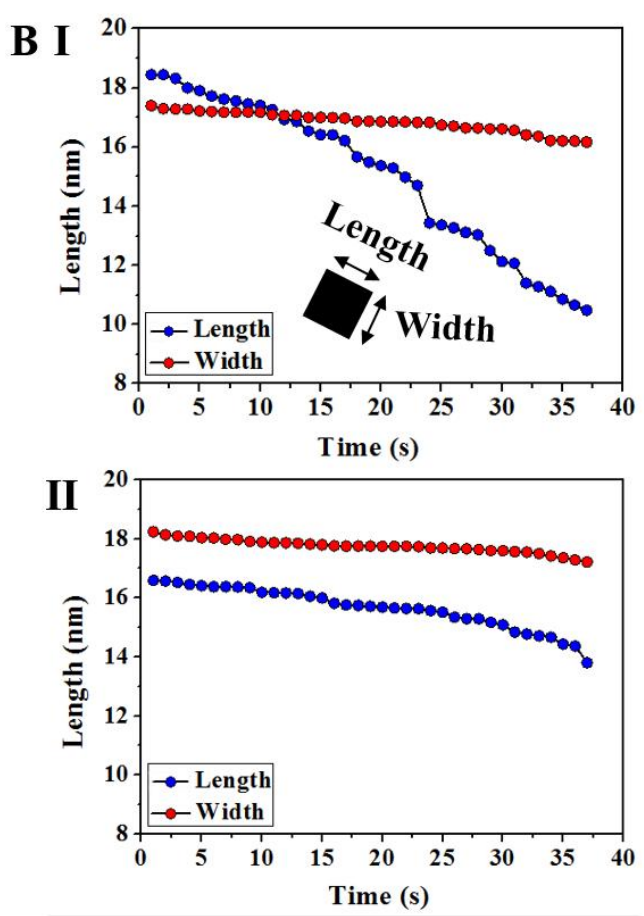
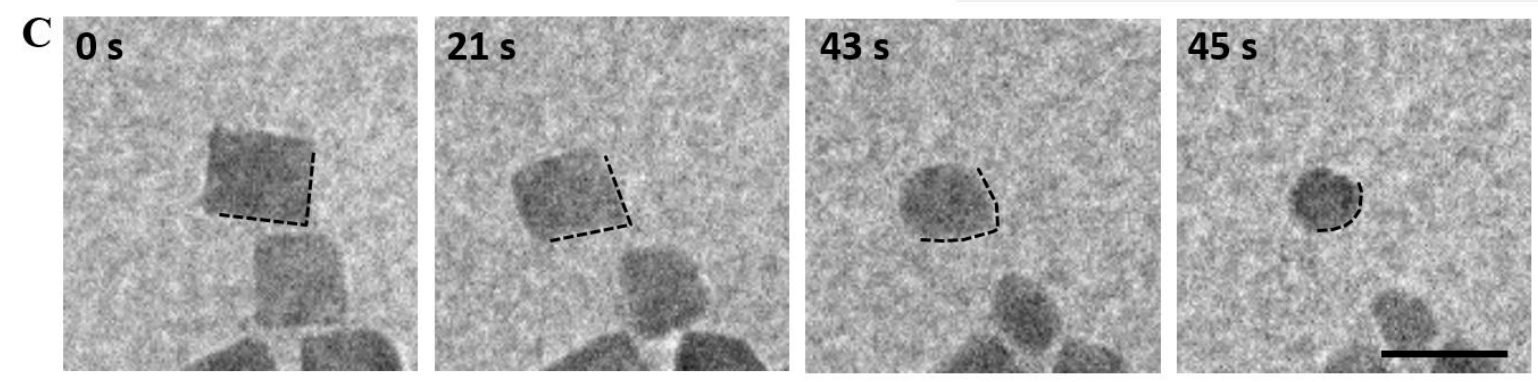

Figure 2. Confinement effect on dissolution kinetics of Pd nanocrystals. A) Sequential TEM images of dissolution kinetics of Pd nanocrystals. B) Changes of lengths and widths of the selected nanocrystals marked as I and II in A as the reaction proceeded. C) The relationship of dissolution kinetics and position with two neighboring nanocrystals. The electron dose used here is 1020 electrons $/ \AA^{2} \cdot$ s. All the scale bars are $20 \mathrm{~nm}$.

TEM observations of the two particles (marked by I and II in Figure 2A) with two and three facets being surrounded by other particles were highlighted and compared. As shown in Figure 2B, the dissolution rate along the 'length' and the 'width' directions from the projection image (inset of Figure 2B) was found to be quite different. For each particle (particles I and II), there was a faster dissolution rate along the 'length' direction (blue line) relative to the 'width' direction (red line). For comparison, these two particles showed the almost same dissolution rate along the 'width' direction, but the extremely different dissolution rate along the length direction (i.e., much faster dissolution rate in particle I 
relative to particle II). All of this difference in the dissolution rate can be attributed to the fact that the slowly dissolving facets is surrounded by other particles, and thus the state of aggregation is important.

This demonstration was also supported by the observation of dissolution process at corners. Similar to the result about the facets, the corners exposed to the reaction solution (marked by black arrows in Figure 2A) solution had a faster dissolution rate relative to the ones surrounded by other particles. Specifically, those exposed corners turned round at the first 17 $\mathrm{s}$, while the others retained their original sharp structure. Figure $2 \mathrm{C}$ shows the dynamic evolution of an individual Pd nanocrystal during dissolution. The marked part of the selected nanocrystal with dotted lines was almost kept intact from $0-21 \mathrm{~s}$, while other three corners exposed to the reaction solution started to be dissolved during this period. The nearest distance of these two integrated nanocrystals is about $1-5 \mathrm{~nm}$ in the first $21 \mathrm{~s}$. When the distance suddenly increased to about $8 \mathrm{~nm}$, the marked part with a sharp corner started to turn round. With the distance further increasing to about $12 \mathrm{~nm}$, the corner disappeared and the marked nanocrystal turned into an isotropic sphere. From this result, we can roughly deduce that the obvious confinement effect for dissolution happens when the nearest distance between two particles is less than $5 \mathrm{~nm}$.

The inhibition of dissolution process for the nanocrystals surrounded by the others is most probably due to the depletion region of the oxidative agent of $\mathrm{Fe}^{3+}$ ions in the narrow and confined space between them. In addition, the diffusion of palladium ions away from the nanocrystal surface into bulk water is inhibited in the confined space, so the high concentration of palladium ions may suppress the dissolution of nanocrystal surface. The similar phenomenon has also been observed in the dissolution of galena nanocrystals in $\mathrm{HCl}$ solution through ex situ TEM analysis (Liu et al., 2008; Liu et al., 2009). The shape transformation of nanocrystals during dissolution with the 2D film is highly influenced by the neighbouring nanocrystals due to the "confinement effect", also known as the "stereo- 
hindrance effect" (Xia et al., 2008; Zhang et al., 2010). It would also have a great influence on the morphologies during growth when the nanocrystals are assembled each other (Liao and Zheng, 2013). This effect happens in many areas such as nanoporous materials (i.e. zeolite) (Kukla et al., 1996), ion transport in biological membranes (Chen and Eisenberg, 1993; Hodgkin and Keynes, 1955; Neher, 1992; Tajkhorshid et al., 2002), catalysis (Lei and Sachtler, 1993), which have received great interest in the last decades. The diffusion coefficient of the species (e.g., molecules and ions) would be severely restrained when the magnitude of their size is commeasurable to the dimension of the restricted geometry (Satterfield et al., 1973).
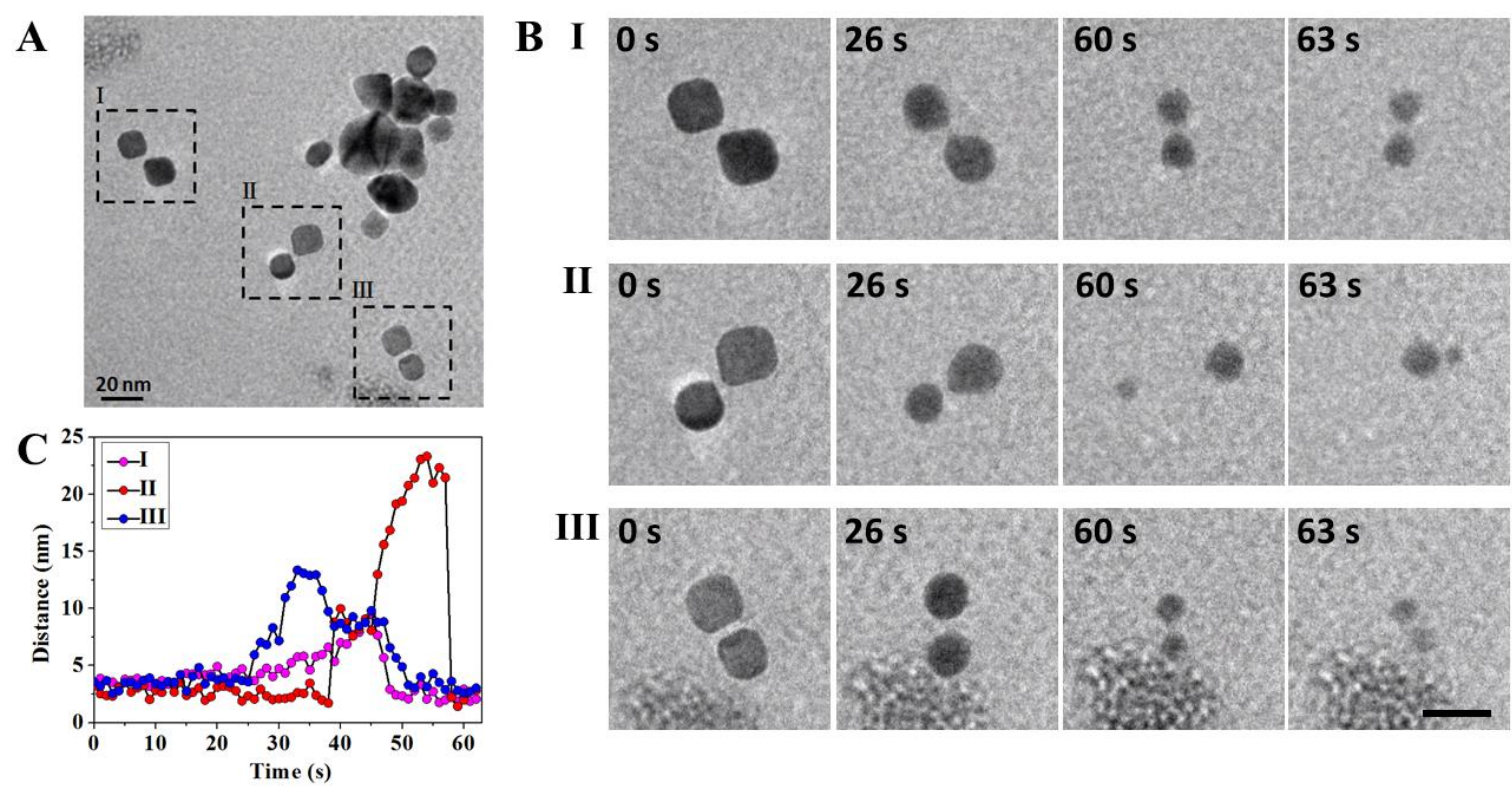

Figure 3. Dynamic activities of three dimers during dissolution. The electron dose used here is 136 electrons/ $\AA^{2} \cdot \mathrm{s}$. A) Overview of nanocrystals at the beginning of dissolution. B) Variation of morphologies and positions of three dimers marked in A during dissolution. The scale bar is $20 \mathrm{~nm}$. C) The distance varieties between two adjacent nanocrystals in three dimers at different times.

We can follow the aggregation kinetics of nanocrystals during dissolution (Chen et al., 1993; Liu et al., 2009). Particles tend to aggregate during dissolution especially when they get smaller. Three dimers were selected here for the detailed observation in Figure 3. Figure 3B 
shows morphology and location evolution of three dimers during dissolution. Figure 3C shows the variation of the nearest distance between two adjacent nanocrystals in dimers during dissolution, and the movement trajectories were depicted in Figure S1. The distances between two nanocrystals in three dimers in the initial stage of the dissolution were all about 3 - $4 \mathrm{~nm}$. But when nanocrystals get smaller during dissolution, the distances between them fluctuated. Nevertheless, two particles in dimer eventually get close with a distance less than $5 \mathrm{~nm}$. For example, two particles kept close at a distance of $2-4 \mathrm{~nm}$ at $0-39 \mathrm{~s}$ in dimer II, one of them suddenly moved to the right side at $40 \mathrm{~s}$. The two particles kept moving, resulting in longer distances. At 50 - $55 \mathrm{~s}$, the distances reached $20-25 \mathrm{~nm}$. But in the next few seconds, the particles aggregated together with a distance of about $2-3 \mathrm{~nm}$. Similar phenomenon happened to the other two dimers. The separation of two particles during dissolution was probably due to the instability of nanocrystals arising from the fast decrease in size. Also, it was probably due to the attraction from the aggregation beside them (see top right corner in Figure 3A). In addition, we notice that two particles kept in a distance of 2-4 $\mathrm{nm}$ apart for most of the time instead of in direct contact. This could be explained by the classic Derjaguin - Landau - Verwey - Overbeek (DLVO) theory (Israelachvili, 2010), which states that the forces between surfaces or particles in liquid can be considered as sum of van der Waals forces and electrostatic forces. Van der Waals forces attract nanocrystals together, and the electrostatic forces make those nanocrystals apart for some distances. Previous result showed that halogen ions like $\mathrm{Cl}^{-}$and $\mathrm{Br}^{-}$ions prefer to absorb on the surface of $\mathrm{Pd}$ nanocrystals, which can make the nanocrystals charged (Chen et al., 2012; Huang et al., 2012; Peng et al., 2013). The $\mathrm{Cl}^{-}$ions here arising from $\mathrm{FeCl}_{3}$ may cause the electrostatic repulsion of Pd nanocrystals from each other. As the rapid change of forces between nanocrystals (red part of the total forces illustrated in Figure S2) including transferring from repulsion to attraction takes place at several or dozens of nanometers scale which was estimated and/or measured by other researchers, the metastable state and the repulsion-attraction behavior all 
happens in similar ranges of distances between nanocrystals (Bowen et al., 1998; Drelich et al., 2006; Ducker et al., 1991; Ducker et al., 1992; Karaman et al., 1993; Liang et al., 2007; Pashley, 1981).

Parallel ex situ experiment with similar experimental conditions were conducted at the room temperature. Nanocrystals with cubic shape enclosed by $\{100\}$ facets (Figure S3) are obtained in the beginning. Most of the Pd nanocrystals were mainly enclosed by low index facets like $\{100\},\{110\}$ and $\{111\}$ during dissolution. The central part of $\{100\}$ faceted was preserved during dissolution, but the corners and edges turned into $\{111\}$ and $\{110\}$ facets gradually. With the same concentration of $\mathrm{Fe}^{3+}$ ions in solution, the dissolution rate is nearly two orders of magnitude faster in liquid cell TEM than in external condition. The average dissolution rate $(d r / d t)$ in liquid cell TEM in Figure 1 is about $0.1 \mathrm{~nm} / \mathrm{s}$ and it is $0.03 \mathrm{~nm} / \mathrm{min}$ in external condition as exhibited in Figure S4. Also, we measured the dissolution rate of nanocrystals in the same solution $\left(0.228 \mathrm{~mol} / \mathrm{L} \mathrm{FeCl}_{3}\right)$ assembled in liquid cell TEM holder (Figure S5). The dissolution rate based on the measured sizes of remaining nanocrystals is $0.03 \mathrm{~nm} / \mathrm{min}$. When the concentration of $\mathrm{FeCl}_{3}$ was reduced to $0.03 \mathrm{~mol} / \mathrm{L}$, the average dissolution rate in the ex situ condition is $0.003 \mathrm{~nm} / \mathrm{min}$ (Figure S6). The reason for the acceleration of dissolution in TEM condition could be the influence of electron-water interaction. Some highly oxidative agents could be generated from electron-water interactions, like $\mathrm{OH} \cdot, \mathrm{HO}_{2} \cdot$, O, $\mathrm{H}_{2} \mathrm{O}_{2}$ and so on (Garrett et al., 2005; Schneider et al., 2014). The standard reduction potential of the primary oxidizing agent $\mathrm{OH} \cdot$ from electron-water interactions is 2.7 $\mathrm{V}$. It is higher than that of $\mathrm{Fe}^{3+}$. However, we have conducted the liquid cell experiment of Pd nanocrystals in $\mathrm{KCl}$ solution instead of $\mathrm{FeCl}_{3}$ solution, no obvious oxidative etching process has been observed. It means that $\mathrm{Fe}^{3+}$ is the dominant oxidizing agent with higher concentration rather than the oxidative species induced from electron-water interactions. However, these agents could participate in the dissolution reaction of $\mathrm{Pd}$ nanocrystals in $\mathrm{FeCl}_{3}$ solution since the higher electron dose would increase the dissolution rate of nanocrystals. 
When the electron dose increases from 136 electrons $/ \AA^{2} \cdot s$ to 1020 electrons $/ \AA^{2} \cdot s$, the average dissolution rate $(d t / d t)$ would change from $0.09 \mathrm{~nm} / \mathrm{s}$ to $0.14 \mathrm{~nm} / \mathrm{s}$ (Figure $\mathrm{S} 7$ ). Besides, the average dissolution rate of nanocrystals in $\mathrm{FeCl}_{3}$ is pretty close to nanocrystals in $\mathrm{KBr}$ as we used in the previous work (Jiang et al., 2014). Nevertheless, the reaction in liquid cell TEM is more complex than that we currently know, more quantitative research is needed from experimental and theoretical approach (Abellan et al., 2014; Hermannsdörfer et al., 2015; Schneider et al., 2014).

\section{Conclusions}

In summary, we have studied the dynamic dissolution of Pd nanocrystals in the presence of $\mathrm{Fe}^{3+}$ ions serving as an oxidative agent using the liquid cell TEM. The dissolution rate of Pd nanocrystals especially for the size less than about $5 \mathrm{~nm}$ was measured from TEM images with high spatial and temporal resolution. We found that the experimental relationship between dissolution rate $(d r / d t)$ and nanocrystal radius $(r)$ agrees well with the prediction of the modified Kelvin equation. A critical size $\left(R_{\text {critical }}\right)$ of around $5 \mathrm{~nm}$ is found in our experimental condition to divide the dissolution kinetics of Pd nanocrystals into two parts. When the nanocrystal size is higher than $R_{\text {critical }}$, the dissolution rate $d r / d t$ is nearly a constant. For the nanocrystal sizing below $R_{\text {critical }}$, the dissolution rate $(d r / d t)$ increases with the decrease of the nanocrystal radius $r$ when the equilibrium solubility is taken into account. Compared to the isolated nanocrystals, the aggregated ones have a dramatically decrease in the dissolution rate due to the confinement effect arising from the depletion of $\mathrm{Fe}^{3+}$ ions in the narrow and confined space. Also, the diffusion of palladium ions in the confined space is inhibited. As a result, the high concentration of palladium ions near particle surface may suppress the dissolution process. In addition, the aggregation kinetics during the dissolution is revealed by monitoring the movement trajectories of the nanocrystals in dimer, which can be interpreted by the balance between van der Waals forces and electrostatic force. This work not 
only greatly advances our understanding of the dissolution kinetics of the nanocrystals, but also provides a fundamental reference for controlled synthesis of nanocrystals in solution.

\section{Acknowledgements}

We acknowledge financial support by the National Basic Research Program of China (Grant No. 2014CB932500 and No. 2015CB921004), the National Science Foundation of China (Grant No. 51472215, No. 51222202, No. 61571197 and No. 61172011). J.Y. acknowledges the EPSRC (UK) funding EP/G070326 and EP/J022098 and supports from Pao Yu-Kong International Foundation for a Chair Professorship in ZJU. The collaboration between C.J. and J.Y. was supported by the 111 project under grant No. B16042. This work made use of the resources of the Center of Electron Microscopy of Zhejiang University.

\section{References}

Abellan, P., Woehl, T.J., Parent, L.R., Browning, N.D., Evans, J.E., Arslan, I., 2014. Factors influencing quantitative liquid (scanning) transmission electron microscopy. Chem. Commun. 50(38), 4873-4880. doi: 10.1039/c3cc48479c.

Asoro, M.A., Kovar, D., Ferreira, P.J., 2013. In situ transmission electron microscopy observations of sublimation in silver nanoparticles. ACS nano 7(9), 7844-7852. doi: $10.1021 / \mathrm{nn} 402771 \mathrm{j}$.

Batchelor, G. K., 2000. An introduction to fluid dynamics, Cambridge University Press, Cambridge.

Bowen, W.R., Hilal, N., Lovitt, R.W., Wright, C.J., 1998. Direct measurement of interactions between adsorbed protein layers using an atomic force microscope. J. Colloid Interface Sci. 197(2), 348-352. doi: 10.1006/jcis.1997.5247.

Chee, S.W., Duquette, D.J., Ross, F.M., Hull, R., 2014. Metastable structures in Al thin films before the onset of corrosion pitting as observed using liquid cell transmission electron microscopy. Microsc. Microanal. 20(2), 462-468. doi: 10.1017/s1431927614000221.

Chen, D.P., Eisenberg, R.S., 1993. Flux, coupling, and selectivity in ionic channels of one conformation. Biophys. J. 65(2), 727-746. doi: 10.1016/s0006-3495(93)81099-3.

Chen, M., Wu, B., Yang, J., Zheng, N., 2012. Small adsorbate-assisted shape control of Pd and Pt nanocrystals. Adv. Mater. 24(7), 862-879. doi: 10.1002/adma.201104145.

Chen, Q., Cho, H., Manthiram, K., Yoshida, M., Ye, X., Alivisatos, A.P., 2015. Interaction potentials of anisotropic nanocrystals from the trajectory sampling of particle motion 
using in situ liquid phase transmission electron microscopy. ACS Cent. Sci. 1(1), 33-39. doi: 10.1021/acscentsci.5b00001.

de Jonge, N., Ross, F.M., 2011. Electron microscopy of specimens in liquid. Nat.

Nanotechnol. 6(11), 695-704. doi:10.1038/nnano.2011.161.

Drelich, J., Long, J., Xu, Z., Masliyah, J., Nalaskowski, J., Beauchamp, R., Liu, Y., 2006. AFM colloidal forces measured between microscopic probes and flat substrates in nanoparticle suspensions. J. Colloid Interface Sci. 301(2), 511-522. doi: 10.1016/j.jcis.2006.05.044.

Ducker, W.A., Senden, T.J., Pashley, R.M., 1991. Direct measurement of colloidal forces using an atomic force microscope. Nature 353(6341), 239.

Ducker, W.A., Senden, T.J., Pashley, R.M., 1992. Measurement of forces in liquids using a force microscope. Langmuir 8(7), 1831-1836.

Evans, J.E., Jungjohann, K.L., Browning, N.D., Arslan, I., 2011. Controlled growth of nanoparticles from solution with in situ liquid transmission electron microscopy. Nano Lett. 11(7), 2809-2813. doi: 10.1021/nl201166k.

Garrett, B.C., Dixon, D.A., Camaioni, D.M., Chipman, D.M., Johnson, M.A., Jonah, C.D., Kimmel, G.A., Miller, J.H., Rescigno, T.N., Rossky, P.J., Xantheas, S.S., 2005. Role of water in electron-initiated processes and radical chemistry: issues and scientific advances. Chem. Rev. 105(1), 355-390. doi: 10.1021/cr030453x.

Goto, K., 1958. Estimation of specific surface area of particles in colloidal silica sols from the rate of dissolution. Bull. Chem. Soc. Jap. 31(8), 900-903. doi: 10.1246/bcsj.31.900.

Hermannsdörfer, J., de Jonge, N., Verch, A., 2015. Electron beam induced chemistry of gold nanoparticles in saline solution. Chem. Commun. 51(91), 16393-16396. doi: 10.1039/c5cc06812f.

Hodgkin, A.L., Keynes, R.D., 1955. The potassium permeability of a giant nerve fibre. J. Physiol. 128(1), 61-88. doi: 10.1113/jphysiol.1955.sp005291.

Huang, X., Li, Y., Li, Y., Zhou, H., Duan, X., Huang, Y., 2012. Synthesis of PtPd bimetal nanocrystals with controllable shape, composition, and their tunable catalytic properties. Nano Lett. 12(8), 4265-4270. doi: 10.1021/nl301931m.

Iler, R. K., 1979. The chemistry of silica: solubility, polymerization, colloid and surface properties and biochemistry of silica, Wiley, New York.

Israelachvili, J. N., 2010. Intermolecular and Surface Forces, Academic Press, California.

Jiang, Y., Zhu, G., Lin, F., Zhang, H., Jin, C., Yuan, J., Yang, D., Zhang, Z., 2014. In situ study of oxidative etching of palladium nanocrystals by liquid cell electron microscopy. Nano Lett. 14(7), 3761-3765. doi: 10.1021/nl500670q.

Jin, M., Liu, H., Zhang, H., Xie, Z., Liu, J., Xia, Y., 2011. Synthesis of Pd nanocrystals enclosed by $\{100\}$ facets and with sizes $<10 \mathrm{~nm}$ for application in CO oxidation. Nano Res. 4(1), 83-91. doi: 10.1007/s12274-010-0051-3.

Karaman, M.E., Meagher, L., Pashley, R.M., 1993. Surface chemistry of emulsion polymerization. Langmuir, 9, 1220-1220.

Kukla, V., Kornatowski, J., Demuth, D., Girnus, I., Pfeifer, H., Rees, L.V.C., Schunk, S., Unger, K.K., Karger, J., 1996. NMR studies of single-file diffusion in unidimensional channel zeolites. Science 272(5262), 702.

Lei, G.D., Sachtler, W.M., 1993. H/D exchange of cyclopentane on Pt/mordenites: Probing for monoatomic Pt sites. J. Catal. 140(2), 601-611. doi: 10.1006/jcat.1993.1109.

Liang, Y., Hilal, N., Langston, P., Starov, V., 2007. Interaction forces between colloidal particles in liquid: Theory and experiment. Adv. Colloid Interface Sci. 134, 151-166. doi: 10.1016/j.cis.2007.04.003.

Liao, H.G., Zheng, H., 2013. Liquid cell transmission electron microscopy study of platinum iron nanocrystal growth and shape evolution. J. Am. Chem. Soc. 135(13), 5038-5043. doi: 10.1021/ja310612p. 
Liao, H.G., Zheng, H., 2016. Liquid cell transmission electron microscopy. Annu. Rev. Phys. Chem. 67, 719-747. doi: 10.1146/annurev-physchem-040215-112501.

Liu, J., Aruguete, D.M., Jinschek, J.R., Rimstidt, J.D., Hochella, M.F., 2008. The nonoxidative dissolution of galena nanocrystals: Insights into mineral dissolution rates as a function of grain size, shape, and aggregation state. Geochim. Cosmochim. Acta 72(24), 5984-5996. doi: 10.1016/j.gca.2008.10.010.

Liu, J., Aruguete, D.M., Murayama, M., Hochella, M.F., 2009. Influence of size and aggregation on the reactivity of an environmentally and industrially relevant nanomaterial (PbS). Environ. Sci. Technol. 43(21), 8178-8183. doi: 10.1021/es902121r.

Ma, Y., Li, W., Zeng, J., McKiernan, M., Xie, Z., Xia, Y., 2010. Synthesis of small silver nanocubes in a hydrophobic solvent by introducing oxidative etching with $\mathrm{Fe}$ (III) species. J. Mater. Chem. 20(18), 3586-3589. doi: 10.1039/c0jm00187b.

Neher, E., 1992. Ion channels for communication between and within cells. Biosci. Rep. 12(1), 1-14. doi:10.1007/bf01125822.

Pashley, R.M., 1981. DLVO and hydration forces between mica surfaces in $\mathrm{Li}^{+}, \mathrm{Na}^{+}, \mathrm{K}^{+}$, and $\mathrm{Cs}^{+}$electrolyte solutions: A correlation of double-layer and hydration forces with surface cation exchange properties. J. Colloid Interface Sci. 83(2), 531-546. doi: 10.1016/00219797(81)90348-9.

Peng, H.C., Xie, S., Park, J., Xia, X., Xia, Y., 2013. Quantitative analysis of the coverage density of $\mathrm{Br}^{-}$ions on $\mathrm{Pd}\{100\}$ facets and its role in controlling the shape of $\mathrm{Pd}$ nanocrystals. J. Am. Chem. Soc. 135(10), 3780-3783. doi: 10.1021/ja400301k.

Ross, F.M., 2015. Opportunities and challenges in liquid cell electron microscopy. Science 350(6267), aaa9886. doi: 10.1126/science.aaa9886.

Ross, F.M., Wang, C., de Jonge, N., 2016. Transmission electron microscopy of specimens and processes in liquids. MRS Bull. 41(10), 791-803. doi: 10.1557/mrs.2016.212.

Satterfield, C.N., Colton, C.K., Pitcher, W.H., 1973. Restricted diffusion in liquids within fine pores. AlChE J. 19(3), 628-635. doi: 10.1002/aic.690190332.

Sau, T. K., Murphy, C. J., 2004. Room temperature, high-yield synthesis of multiple shapes of gold nanoparticles in aqueous solution. J. Am. Chem. Soc. 126(28), 8648-8649. doi: 10.1021/ja047846d.

Schneider, N.M., Norton, M.M., Mendel, B.J., Grogan, J.M., Ross, F.M., Bau, H.H., 2014. Electron-water interactions and implications for liquid cell electron microscopy. J. Phys. Chem. C 118(38), 22373-22382. doi: 10.1021/jp507400n.

Tajkhorshid, E., Nollert, P., Jensen, M.Ø., Miercke, L.J., O'connell, J., Stroud, R.M., Schulten, K., 2002. Control of the selectivity of the aquaporin water channel family by global orientational tuning. Science, 296(5567), 525-530. doi: 10.1126/science.1067778.

Tao, A.R., Habas, S., Yang, P., 2008. Shape control of colloidal metal nanocrystals. Small 4(3), 310-325. doi: 10.1002/smll.200701295

Wang, X., Zhuang, J., Peng, Q., Li, Y., 2005. A general strategy for nanocrystal synthesis. Nature 437, 121-124. doi:10.1038/nature03968.

Weast, R.C., 1980. Handbook of chemistry and physics 60th Edition, CRC Press, Florida.

Williamson, M.J., Tromp, R.M., Vereecken, P.M., Hull, R., Ross, F.M., 2003. Dynamic microscopy of nanoscale cluster growth at the solid-liquid interface. Nat. Mater. 2(8), 532-536. doi: 10.1038/nmat944.

Wu, J., Shan, H., Chen, W., Gu, X., Tao, P., Song, C., Shang, W., Deng, T., 2016. In situ environmental TEM in imaging gas and liquid phase chemical reactions for materials research. Adv. Mater. 28(44), 9686-9712. doi: 10.1002/adma.201602519.

Xia, Q., Chen, X., Zhao, K., Liu, J., 2008. Synthesis and characterizations of polycrystalline walnut-like CdS nanoparticle by solvothermal method with PVP as stabilizer. Mater. Chem. Phys. 111(1), 98-105. doi: 10.1016/j.matchemphys.2008.03.020. 
Xia, Y., Xiong, Y., Lim, B., Skrabalak, S.E., 2008. Shape-controlled synthesis of metal nanocrystals: simple chemistry meets complex physics? Angew. Chem. Int. Ed. 48(1), 60-103. doi: 10.1002/anie.200802248.

Xiong, Y., Chen, J., Wiley, B., Xia, Y., Aloni, S., Yin, Y., 2005. Understanding the role of oxidative etching in the polyol synthesis of Pd nanoparticles with uniform shape and size. J. Am. Chem. Soc. 127(20), 7332-7333. doi: 10.1021/ja0513741.

Xiong, Y., Chen, J., Wiley, B., Xia, Y., Yin, Y., Li, Z.Y., 2005. Size-dependence of surface plasmon resonance and oxidation for Pd nanocubes synthesized via a seed etching process. Nano Lett. 5(7), 1237-1242. doi: 10.1021/nl0508826.

Zeng, Z., Zhang, X., Bustillo, K., Niu, K., Gammer, C., Xu, J., Zheng, H., 2015. In situ study of lithiation and delithiation of $\mathrm{MoS}_{2}$ nanosheets using electrochemical liquid cell transmission electron microscopy. Nano Lett. 15(8), 5214-5220. doi: 10.1021/acs.nanolett.5b02483.

Zhang, H., Jin, M., Wang, J., Li, W., Camargo, P.H., Kim, M.J., Yang, D., Xie, Z., Xia, Y., 2011. Synthesis of Pd-Pt bimetallic nanocrystals with a concave structure through a bromide-induced galvanic replacement reaction. J. Am. Chem. Soc. 133(15), 6078-6089. doi: 10.1021/ja201156s.

Zhang, H., Jin, M., Xia, Y., 2012. Noble-metal nanocrystals with concave surfaces: synthesis and applications. Angew. Chem. Int. Ed. 51(31), 7656-7673. doi: 10.1002/anie.201201557.

Zhang, L., Roling, L.T., Wang, X., Vara, M., Chi, M., Liu, J., Choi, S.I., Park, J., Herron, J.A., Xie, Z., Mavrikakis, M., Xia. Y., 2015. Platinum-based nanocages with subnanometerthick walls and well-defined, controllable facets. Science 349(6246), 412-416. doi: 10.1126/science.aab0801.

Zhang, Y., Xu, J., Xu, P., Zhu, Y., Chen, X., Yu, W., 2010. Decoration of ZnO nanowires with Pt nanoparticles and their improved gas sensing and photocatalytic performance. Nanotechnology 21(28), 285501. doi: 10.1088/0957-4484/21/28/285501.

Zheng, H., Smith, R.K., Jun, Y.W., Kisielowski, C., Dahmen, U., Alivisatos, A.P., 2009. Observation of single colloidal platinum nanocrystal growth trajectories. Science 324(5932), 1309-1312. doi: 10.1126/science.1172104.

Zheng, Y., Zeng, J., Ruditskiy, A., Liu, M., Xia, Y., 2013. Oxidative etching and its role in manipulating the nucleation and growth of noble-metal nanocrystals. Chem. Mater. 26(1), 22-33. doi: 10.1021/cm402023g.

Zhu, G., Jiang, Y., Huang, W., Zhang, H., Lin, F., Jin, C., 2013. Atomic resolution liquid-cell transmission electron microscopy investigations of the dynamics of nanoparticles in ultrathin liquids. Chem. Comm. 49(93), 10944-10946. doi: 10.1039/c3cc46667a. 